\title{
Study of the structures of the explosive events in the UV
}

\author{
R. T. Niembro-Hernandez ${ }^{1}$, J. E. Mendoza-Torres ${ }^{2}$ and K. Wilhelm ${ }^{3}$ \\ ${ }^{1}$ Fac. de Ciencias, UNAM, Mexico \\ email: tat17v@gmail.com \\ ${ }^{2}$ Instituto Nacional de Astrofisica, Optica y Electronica, INAOE, Mexico \\ email: mend@inaoep.mx \\ ${ }^{3}$ Max-Planck-Institute for Solar System Research, MPS, Germany \\ email: wilhelm@linmpi.mpg.de
}

\begin{abstract}
We analyze Si IV $139.37 \mathrm{~nm}$ emission line during solar Explosive Events (EE) near the center of the solar disk with the aim to study the structure of the sources of EEs observed at the VUV. The observations were made by SUMER, on board SoHO, with a raster regime of six EW positions that allowed us to identify the times and EW, NS positions of the maximum amplitude of each EE. Based on one dimensional NS distributions at three different wavelengths (blue, central and red) for times around each maximum we have identified three different shapes of the sources. Also, It was found that the maximum at the blue wing is attained after the maxima at red and central wavelengths.
\end{abstract}

Keywords. Sun: UV radiation, corona, activity, techniques: spectroscopic

\section{Introduction}

A class of some sudden increase of VUV emission in small spatial scale $(2-4$ arcsec) that take place at the solar TR are referred to as Explosive Events (EEs). EEs occur in $1-4$ minutes (Kirk et al. 1994), sometimes with line wing increases that denote flows of $100-250 \mathrm{~km} / \mathrm{s}$ (Innes \& Toth 1999).

EEs have been observed with a rate of occurrence of more than 1000 per second all over the solar disk (Brueckner \& Bartoe 1983) which could give a non negligible input of energy for Coronal Heating.

The extension of the EEs has been studied considering the region where the amplitude of the area covered by the slit was maximum, but it has not been verified if the observed increase corresponds to the maximum attained over the extension of the EE (MendozaTorres, Torres-Papaqui \& Wilhelm 2005).

In studying the structure (projected on the disk) of EEs it is important to know the actual extension. In order to do this, it is necessary to identify the events that really where sampled all the way during the increasing and decreasing phases and whose maximum became at the central regions of the observed field. Then, we are able to study the structure over such field.

A better knowledge of the source structure can help us to identify the energy transport between the layers of the Sun around the TR, (Chae et al. 1998a).

\section{Observations}

We selected SUMER data for the Si IV line at $139.37 \mathrm{~nm}$ (Wilhelm et al. 1995 in a raster regimen that produces spectra for a field of $120^{\prime \prime} \mathrm{NS} \times 6^{\prime \prime} \mathrm{EW}$ of June 20th 


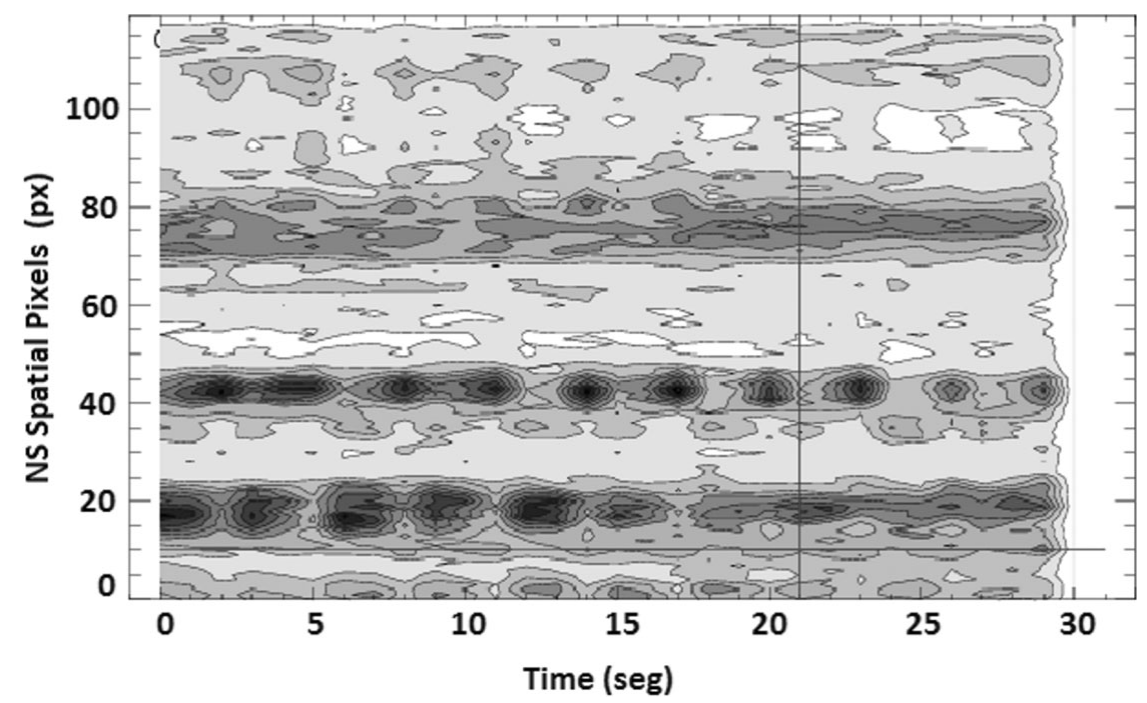

Figure 1. Plot of amplitude as function of NS position and time. It may be seen that EEs take place at some preferential locations.

1996, where Coronal Holes and Active Regions were not observed, with an integration of 5 seconds at each position.

\section{Data analysis and results}

From the $2.5 \mathrm{~h}$ of SUMER data for June 20th, 1996 we selected EEs based on some criteria. In such a way we selected EEs for which both the rising and the decreasing phases were observed and whose maximum was not observed at the extremes of the field. With these restrictions we get a sample of 41 EEs (Table 1). For the selected EEs, one dimensional NS distribution profiles were made for the amplitude at the central wavelength of the line and for wavelengths at both wings (blue and red) for times around the maximum. This method does not require to fit Gaussians in order to identify flows. We have seen that EEs are observed at some given preferential locations (Fig. 1).

The extensions of the sources during the maximum at each of the wavelengths were estimated at 0.5 and at 0.2 of the maximum amplitude.

Table 1. Amount of EEs at the different wavelengths and combinations of them

\begin{tabular}{lccc}
\hline Component & Amount & Combination & Amount \\
\hline All & 41 & Blue-Central-Red & 18 \\
& & Blue-Central & 2 \\
Blue & 26 & Blue-Red & 5 \\
& & Central-Red & 5 \\
Central & 26 & Blue & 1 \\
\multirow{2}{*}{ Red } & \multirow{2}{*}{33} & Central & 1 \\
& & Red & 5 \\
\hline
\end{tabular}

Based on the NS distributions we noticed that one after another maxima can take place around a given location which means that an EE could be related to more than one 
energy release. Most of the EEs have a component of low amplitude but large extension sorrounding the maximum amplitude as shown in Fig. 2c.

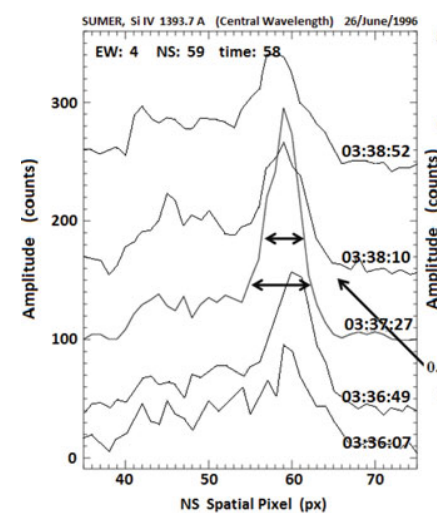

a)

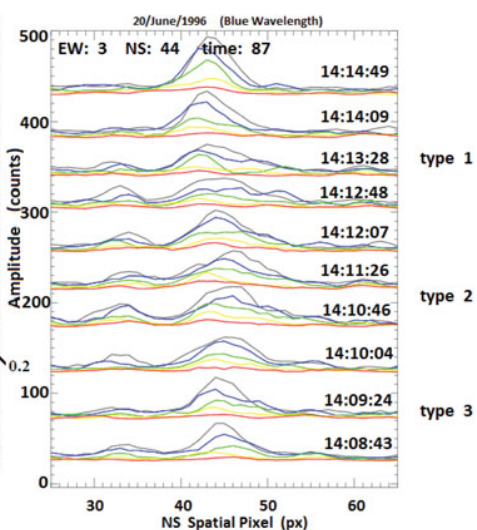

b)

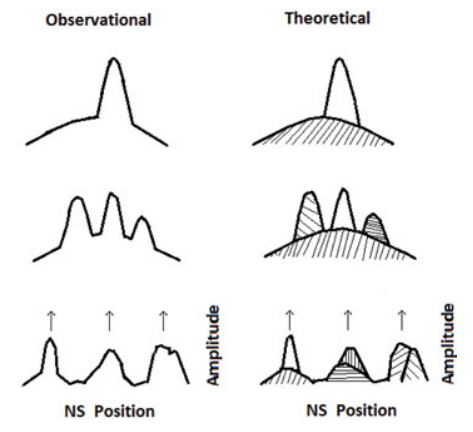

c)

Figure 2. a) NS Distributions for the growing, the maximum and the decreasing phase of an EE. An extended region with increased amplitude is observed during the EE, local variations at this region occur during the EE. b) Amplitude distributions around the time and location of the maximum amplitude of an EE. Increases at near locations are seen. c) Schematic representation of the possible sources that could be taking place for the different types of distributions observed to occur in the studied EEs.

We found out that $18 \mathrm{EE}$ showed increases of the three wavelengths and fulfilled the selection criteria.

Expansion and displacement of the sources are sometimes seen after the occurrence of the maximum amplitude (Fig. 2b).

In most of the cases the blue maxima occurred after the central maxima (11 cases of $18 \mathrm{EEs})$. For the red component we found that in 7 of 18 ocassions it took place as the central maxima (at the same time) and 4 of 18 red was attained before the central maxima (Table 1$)$.

This behaviour and the spatial one (Fig. 2) around maxima seem to indicate that more than one energy releases take place or that it happens several up and down flows in one $\mathrm{EE}$, possible in different locations and in different phases of the EE.

\section{References}

Innes, D. E. \& Toth, G. 1999, Solar Physics, vol. 185, 127-141

Wilhelm, K. et al. 1995, Solar Physics, vol. 162, 189-231

Chae et al. 2003, Journal of the Korean Astronomical Society, vol. 36, 13-20

Brueckner, G. E. \& Bartoe J.-D. F. 1983, Astrophysical Journal, vol. 272, 329-348

Chae, J., Wang, H., Lee, C., Goode, P., \& Schuhle, U. 1998, Astrophysical Journal, vol. 504, 123

Mendoza-Torres, J. E., Torres-Papaqui, J. P., \& Wilhelm, K. 2005, Astronomy and Astrophysics, vol. 431, 339-244

Brekke, P., Hassler, D. M., \& Wilhelm, K. 1997, Solar Physics, vol. 175, 349-374

Dere, K. P. 1994, Advances in Space Research, vol. 14, 413-422 